INPLASY

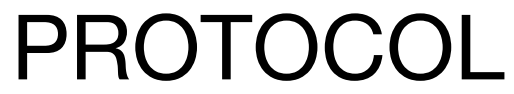

To cite: Ramirez-Campillo.

Exercise-induced spot reduction: a systematic review with meta-analysis. Inplasy protocol 202160103. doi: 10.37766/inplasy2021.6.0103

Received: 28 June 2021

Published: 28 June 2021

Corresponding author:

Rodrigo Ramirez-Campillo

r.ramirez@ulagos.cl

Author Affiliation:

Universidad de Los Lagos

Support: None.

Review Stage at time of this submission: Preliminary searches.

Conflicts of interest:

None declared.

\section{Exercise-induced spot reduction: a systematic review with meta-analysis}

Ramirez-Campillo, R¹.

Review question / Objective: To summarize the peer-review literature assessing the effects of exercise on spot reduction. Condition being studied: Spot reduction. Information sources: Pubmed, Scopus, WOS, reference lists from included studies, external experts.

INPLASY registration number: This protocol was registered with the International Platform of Registered Systematic Review and Meta-Analysis Protocols (INPLASY) on 28 June 2021 and was last updated on 28 June 2021 (registration number INPLASY202160103).

\section{INTRODUCTION}

Review question / Objective: To summarize the peer-review literature assessing the effects of exercise on spot reduction.

Condition being studied: Spot reduction.

\section{METHODS}

Participant or population: Healthy participants, without restriction on age or sex.
Intervention: Unilateral exercise training.

Comparator: Contralateral limb.

Study designs to be included: Controlled trials.

Eligibility criteria: According to the PICOS approach. 
Information sources: Pubmed, Scopus, WOS, reference lists from included studies, external experts.

Main outcome(s): Fat-related measures (other than intra-muscular) from trained and non-trained limbs.

Additional outcome(s): Potential adverse effects (e.g., injury).

Quality assessment / Risk of bias analysis: PEDro scale.

Strategy of data synthesis: Pre- and postintervention mean \pm standard deviation (SD) for a given fat-related outcome from the trained and control groups were converted to Hedges' g effect size (ES). A meta-analysis for a given fat-related outcome was conducted if at least three studies provided sufficient data for the calculation of ES. The data were standardized using post score SD. For studies that reported standard errors, standard deviations were calculated by multiplying the standard error with the square root of the sample size. In all analyses, we used the random-effects model to account for differences between studies that might impact the treatment effect. The ES values are presented alongside their respective $95 \% \mathrm{Cls}$. Calculated ES were interpreted using the following scale: $<0.2$, trivial; $0.2-0.6$, small; > 0.6 - 1.2, moderate; > 1.2 - 2.0, large; > 2.0 - 4.0, very large; > 4.0, extremely large. Heterogeneity was assessed using the $\mathbf{1 2}$ statistic. $\mathbf{2}$ values of $<25 \%, 25-75 \%$, and $>75 \%$, were considered to represent low, moderate and high levels of heterogeneity, respectively. The risk of bias was explored using the extended Egger's test. All analyses were carried out using the Comprehensive MetaAnalysis program (version 2; Biostat, Englewood, NJ, USA). The statistical significance threshold was set at $p<0.05$.

Subgroup analysis: In addition to the main analyses, we used the median split technique to explore subgroup analyses to determine the influence of training programme duration, type of exercise (e.g., resistance training vs. cycling), and the total number of training sessions. We also performed subgroup analyses according to participants' age, and sex (male vs. female). Additional subgroup also considered the type of measurement instrument (e.g., callipers vs. MRI), and anatomical point of measurement (e.g., leg vs. arm).

Sensitivity analysis: Not planned a priory

Language: No limitation on language.

Country(ies) involved: Chile.

Keywords: exercise; human physical conditioning; resistance training; highintensity interval training; body composition; subcutaneous fat.

Contributions of each author:

Author 1 - Rodrigo Ramirez-Campillo. 